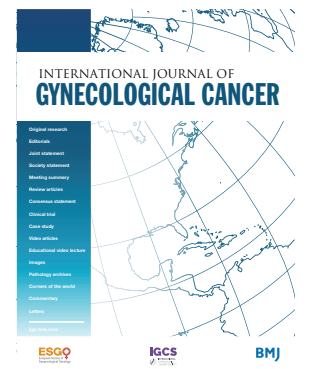

${ }^{1}$ Kelly Gynecologic Oncology Division, Department of Gynecology and Obstetrics, Johns Hopkins University School of Medicine, Baltimore, Maryland, USA

${ }^{2}$ Division of Gynecologic Oncology, Department of Obstetrics and Gynecology, University of Minnesota, Minneapolis, Minnesota, USA ${ }^{3}$ TeLinde Gynecologic Pathology Program, Department of Gynecology and Obstetrics, Johns Hopkins University School of Medicine, Baltimore, Maryland, USA

\section{Correspondence to} Dr J Stuart Ferriss, Department of Gynecology and Obstetrics, Johns Hopkins University, Baltimore, MD 21218, USA; jferris3@jhmi.edu

Received 6 May 2021 Accepted 21 June 2021 Published Online First 1 July 2021

\title{
Uterine serous carcinoma: key advances and novel treatment approaches
}

\author{
J Stuart Ferriss (D), ${ }^{1}$ Britt K Erickson, ${ }^{2}$ le-Ming Shih, ${ }^{3}$ Amanda N Fader ${ }^{1}$
}

\begin{abstract}
The incidence and mortality rates from endometrial cancer continue to increase worldwide, while rates in most other cancers have either plateaued or declined considerably. Uterine serous carcinoma represents a fraction of all endometrial malignancies each year, yet this histology is responsible for nearly $40 \%$ of all endometrial cancerrelated deaths. These deaths disproportionately affect black women, who have higher rates of advanced disease at diagnosis. Molecular genetic analyses reveal major alterations including TP53 mutation, PIK3CA mutation/ amplification, ERBB2 amplification, CCNE1 amplification, FBXW7 mutation/deletion, PPP2R1A mutation, and somatic mutations involving homologous recombination genes. Clinical risk factors for uterine serous carcinoma include advancing age, a history of breast cancer, tamoxifen usage, and the hereditary breast-ovarian cancer syndrome. Surgery remains the cornerstone of treatment. Recent advances in our understanding of uterine serous carcinoma molecular drivers have led to development of targeted therapeutics that promise improved outcomes for patients. Overexpression or amplification of HER2 in uterine serous carcinoma carries a poor prognosis; yet this actionable target has led to the incorporation of several anti-HER2 therapies, including trastuzumab which, when added to conventional chemotherapy, is associated with improved survival for women with advanced and recurrent HER2-positive disease. The combination of pembrolizumab and lenvatinib is also a promising targeted treatment strategy for women with uterine serous carcinoma, with a recent phase II study suggesting a $50 \%$ response rate in women with recurrent disease. Several trials examining additional targeted agents are ongoing. Despite years of stalled progress, meaningful, tailored treatment options are emerging for patients with this uncommon and biologically aggressive endometrial cancer subtype.
\end{abstract}

\section{INTRODUCTION}

Uterine serous carcinoma is an uncommon cancer that continues to have a significant effect on patient outcomes and survival. High rates of extra-uterine disease at diagnosis, combined with a paucity of effective therapies, have limited our ability to make meaningful progress in the treatment of women with uterine serous carcinoma. With an improved understanding of molecular drivers, recent targeted therapeutic advances have led to improved outcomes in those with both advanced and recurrent disease. Herein, we review the epidemiologic trends, pathobiology, and molecular drivers of uterine serous carcinoma; critically appraise the evidence supporting best surgical, chemotherapeutic, and targeted therapy practices; and discuss targeted therapy trials as well as contemporary treatment recommendations.

\section{EPIDEMIOLOGY AND DISPARITIES}

Endometrial cancer is one of the most common cancers diagnosed in women and is one of the few malignancies for which both incidence and mortality continue to increase. ${ }^{12}$ Uterine serous carcinoma is a high-grade endometrial cancer subtype that represents approximately $5-10 \%$ of all endometrial malignancies cases each year. ${ }^{34}$ Despite representing a small proportion of endometrial cancer cases, uterine serous carcinoma accounts for an alarming 39\% of disease-specific deaths. Features of uterine serous carcinoma that support this observation include higher rates of deep myometrial invasion, as well as metastatic spread to both lymph nodes and peritoneal surfaces compared with endometrioid tumors. ${ }^{3}$

These poor outcomes disproportionately affect black women, who are more likely to be diagnosed with uterine serous carcinoma (26\%) compared with both Hispanic (18.6\%) and non-Hispanic white women $(16.6 \%) .{ }^{5}$ Black women are more likely to present with an advanced-stage, high-grade endometrial cancer and are $21 \%$ more likely to die of the disease than both Hispanic and non-Hispanic white women. ${ }^{2}$ Black women with high-grade carcinoma have been found to be less likely to be treated with surgery and more likely to receive chemotherapy alone compared with other groups. ${ }^{5}$

\section{Risk Factors}

Specific risk factors for uterine serous carcinoma include a personal history of breast cancer, tamoxifen exposure, and hereditary cancer syndromes. Common clinical features include older age and higher stage at diagnosis, and mutations in p53.

\section{Breast Cancer and Tamoxifen}

There appears to be a connection between use of tamoxifen after breast cancer and subsequent development of all endometrial cancer subtypes. The National Surgical Adjuvant Breast and Bowel Project (NSABP) protocol B-14 randomized node-negative patients with breast cancer to adjuvant therapy with 
either placebo or tamoxifen. ${ }^{6}$ The tamoxifen arm had 15 endometrial cancers compared with 0 in the placebo arm $(p<0.001)$. Three of the $15(20 \%)$ endometrial cancers among women randomized to tamoxifen were uterine serous carcinoma. Although this protocol was not designed to investigate the rate of endometrial cancer in this population, a $20 \%$ rate of serous carcinoma is higher than would have been expected. Gehrig et al analyzed 1166 women with endometrial cancer, of whom $54(5 \%)$ also had a prior history of breast cancer. ${ }^{7}$ The authors noted a 2.6 -fold increase in the diagnosis of uterine serous carcinoma among women with a history of breast cancer in this single institution study. However, an association between tamoxifen use and a specific uterine histology was not observed and the study was likely underpowered to answer this question. Another single institution study evaluated women with endometrial cancer and found $8 \%$ had a history of breast cancer, half of whom had taken tamoxifen. ${ }^{8}$ The authors observed that women with a history of breast cancer had higher rates of highgrade endometrial cancers than those without such a history ( $31 \%$ vs $18 \%, p=0.02$ ).

\section{Hereditary Cancer Syndromes}

A major limitation of the retrospective reviews evaluating the association between breast cancer and high-grade endometrial cancer was that rates of BRCA mutations were not reported. A link between uterine serous carcinoma and the hereditary breast-ovarian cancer syndrome via mutations in the BRCA family of proteins has been proposed. ${ }^{9}$ Lavie et al studied Israeli women with uterine serous carcinoma to determine if there is an association with BRCA mutations. ${ }^{10-12}$ Their estimates of the rate of BRCA mutations among patients with uterine serous carcinoma have ranged from $15 \%$ to $27.3 \%$. A separate multi-institutional series of 151 patients with uterine serous carcinoma estimated the rate of BRCA mutation to be $5 \% .{ }^{13}$ Among patients with BRCA mutations, an international prospective study found higher observed to expected ratio of uterine serous carcinoma (observed:expected ratio $22.2,95 \% \mathrm{Cl} 6.1$ to 56.9; $\mathrm{p}<0.001) .{ }^{14}$ Laitman et al reported a retrospective casecontrol study evaluating 2627 Jewish BRCA mutation carriers and 1844 age and ethnicity-matched controls. ${ }^{15}$ They found a higher rate of uterine serous carcinoma in the BRCA mutation carriers than in the control population (observed:expected ratio $14.3 ; 95 \% \mathrm{Cl} 4.6$ to $33.3 ; p<0.001$ ). The authors concluded that current recommendations of risk-reducing bilateral salpingo-oophorectomy do not need to be modified to include hysterectomy given the low lifetime risk of death from endometrial cancer in the general population; however, selected patients of Ashkenazi Jewish descent may wish to consider the role of hysterectomy in this setting.

Hereditary non-polyposis colonic carcinoma, or Lynch syndrome, carries a lifetime risk of uterine cancer 30-60 times higher than the general population. Pennington et al evaluated a series of 151 uterine serous carcinoma tumors from several institutions to estimate the rate of mismatch repair defects in this setting. ${ }^{13}$ They did not identify any patients who met criteria for the diagnosis of Lynch syndrome and concluded that mismatch repair defects are rare in uterine serous carcinoma.

\section{Prognosis}

The prognosis for uterine serous carcinoma compared with more common endometrial cancer types is generally poor. The 5 -year



Figure 1 Gross and microscopic features of uterine serous carcinoma. (A) The $7 \mathrm{~cm}$ tumor mass occupies the posterior uterine cavity. (B). Histology shows the characteristic papillary architecture with highly atypical tumor cells. (C). A high-magnification view reveals invasion of the tumor into a blood vessel. The carcinoma cells are high grade and an abnormal mitotic figure (arrow) is evident.

overall survival for stage I uterine serous carcinoma is driven by depth of myometrial invasion. Non-invasive uterine serous carcinoma has an estimated $90 \% 5$-year overall survival compared with minimally invasive ( $<50 \%$ myometrial invasion) cancers at $80 \%$, and deeply invasive ( $>50 \%$ myometrial invasion) cancers at $66 \%$. The combined 5-year overall survival estimate for stage I-II disease is $74 \%$ compared with $92.7 \%$ for endometrioid cancers. Stage III-IV serous cancers have a $33 \% 5$-year overall survival. ${ }^{3}$

\section{Pathogenesis and Molecular Biology}

Microscopically, uterine serous carcinomas have a predominate papillary pattern and can also have more solid areas with slit-like glandular spaces ${ }^{4}$ (Figure 1). Up to one-third of cases can be of mixed histology with varying amounts of endometrioid or clear cell carcinoma. Pathologists are often confronted with the need to classify these tumors despite seemingly divergent microscopic findings. Immunohistochemical stains are frequently used to further categorize tumors when histology alone is unclear. One of the often-cited features of uterine serous carcinoma on immnunohistochemistry is abnormal (mutated) p53 staining. Although p53 staining, especially the pattern (diffuse and intense immunoreactivity) compatible with missense TP53 mutations, is a useful marker to support the diagnosis of uterine serous carcinoma. Notably, high-grade endometrioid carcinoma and high-grade serous carcinomas of the fallopian tube and ovary also show the same staining pattern. Diffuse p16 and minimum WT-1 immunostaining are also frequently used in pathology as adjuncts to assist the differential diagnosis in difficult cases. ${ }^{16}$

Nearly $90 \%$ of cases can be associated with the proposed precursor lesion, endometrial intraepithelial carcinoma. Occasionally, endometrial intraepithelial carcinoma can be seen in the 


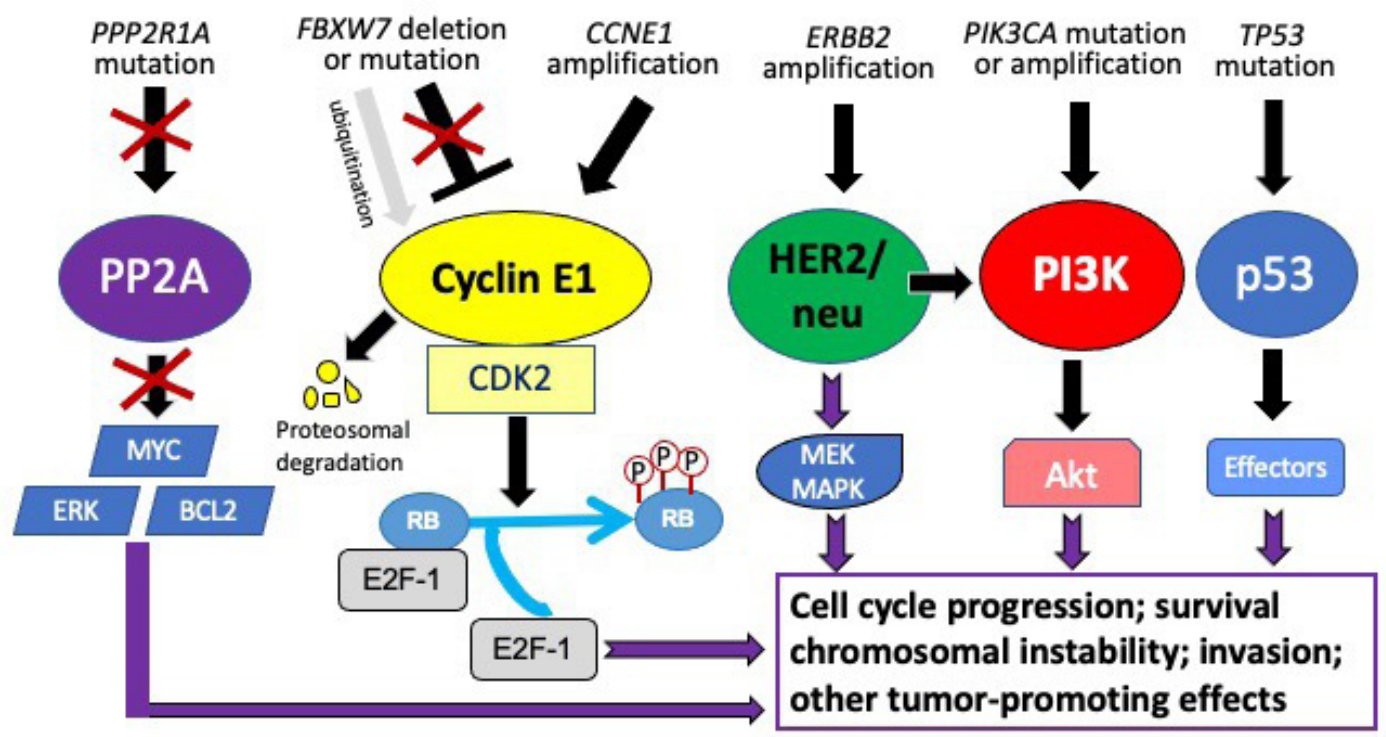

Figure 2 The major molecular genetic alterations in uterine serous carcinoma. Molecular analyses have identified six major somatic genetic alterations in uterine serous carcinomas: TP53 mutation, PIK3CA activating mutation and its gene amplification, ErBB2 gene amplification, CCNE1 gene amplification, FBXW7 deletion or inactivating mutation, and PPP2R1A inactivating mutation. These genetic alterations cause aberration of downstream pathway activities and signaling transduction cascades, leading to enhanced cellular proliferation and cell survival, chromosomal instability, and tumor invasion. These pathways also offer new opportunities for targeted intervention.

absence of definitive uterine serous carcinoma or with carcinoma confined to the endometrium. Endometrial intraepithelial carcinoma and uterine serous carcinoma can often arise from an endometrial polyp in a background of atrophic endometrium. Even non-invasive endometrial intraepithelial carcinoma can be associated with lymphovascular invasion and therefore risk of metastasis. In these instances, surgical staging is required, given reports of metastatic disease documented with a complete surgical evaluation. ${ }^{17}$

Comprehensive genome-wide analyses have established a firmer understanding of the relevant pathogenic pathways. ${ }^{18}$ These studies demonstrated that uterine serous carcinoma is characterized by six major molecular genetic alterations including: TP53 mutation, PIK3CA activating mutation/amplification, ERBB2 amplification, CCNE1 amplification, FBXW7 inactivating mutation/deletion and PPP2R1A inactivating mutation (Figure 2). Many of the molecular genetic alterations, including TP53 mutations, PIK3CA mutation, FBXW7 mutation, and CCNE1 amplification, found in uterine serous carcinoma are readily detected in endometrial intraepithelial carcinoma, suggesting that these genetic events occur early during carcinogenesis. ${ }^{19}$

\section{Molecular Classification and Actionable Molecular Targets}

The molecular characterization of endometrial cancer performed by the Cancer Genome Atlas (TCGA) consortium defined four prognostic subgroups: the POLE cohort; microsatellite instable cohort; and the copy number low and copy number high cohorts. Uterine serous carcinoma tumors are considered copy number high tumors due to their extensive copy number alterations. ${ }^{20}$ The Proactive Molecular Risk Classifier for Endometrial Cancer (ProMisE) was created as a more practical classification of endometrial cancers also with four categories: POLE, microsatellite instable, p53 mutant, and 553 wild type. ${ }^{21}$ Uterine serous carcinoma tumors fall in the p53 mutant group.
It has been suggested that the response of the immune system (presence or absence of tumor infiltrating lymphocytes), rather than molecular subtype alone, may offer a useful clinical classification. ${ }^{22}$ The presence of tumor infiltrating lymphocytes in the tumor microenvironment signal a recognition by the immune system-thus marking them as potential responders to checkpoint inhibition. This is regardless of microsatellite stable or mutated p53 status, which means that some of these patients may still respond to immunotherapy.

\section{Expression of HER2}

HER2/neu is a receptor tyrosine-protein kinase erbB-2 that is encoded by the ERBB2 gene. It is expressed in a variety of normal tissues and mediates proliferation, differentiation, and apoptosis. ${ }^{23}$ In the last decade, HER2 was recognized as an important target in uterine serous carcinoma, expressed or amplified in approximately $20-40 \%$ of both early-stage and advanced-stage tumors. ${ }^{24} 25$ HER2 expression or amplification in uterine serous carcinoma is associated with worse prognosis. A recent study demonstrated the prognostic potential of HER2 in uterine serous carcinoma. In that study, patients with HER2-positive stage I tumors were three times more likely to have a recurrence of disease than those with HER2negative tumors, even when treated with adjuvant therapy after hysterectomy. ${ }^{25}$ Given high rates of HER2 expression in uterine serous carcinoma, and evidence that HER2-positive tumors correlate with worse outcomes, there is a rationale of therapeutically targeting HER2 in uterine serous carcinoma.

Algorithms exist for scoring HER2 expression and amplification in breast and gastric carcinomas; however, validated criteria do not exist for endometrial cancers. In clinical practice, the American Society of Clinical Oncology/College of American Pathologists algorithms for breast cancer are used. ${ }^{26}$ Tumors are first evaluated for protein expression with an immunohistochemistry assay. 
Results are reported on a scale of $0-3$. Cases that are 0 or $1+$ are considered negative for HER2 expression. Cases that are $3+$ are considered positive. If the scoring is $2+$ (defined as incomplete/ weak staining in $>10 \%$ of tumor cells or complete/intense staining in $<10 \%$ of tumor cells), the tumor is then tested using in situ hybridization. However, in a study from Johns Hopkins examining the concordance of immunohistochemistry and fluorescent in situ hybridization (FISH) in uterine serous carcinoma and serous endometrial intraepithelial carcinoma specimens, $14 \%$ of cases reported as 0/1+ HER2 were FISH amplified. ${ }^{27}$ Therefore, we recommend that both immunohistochemistry and FISH be performed on uterine serous carcinoma until clinical trials correlate HER2 status with clinical response to HER2-targeted therapy.

\section{Defects in Homologous Recombination}

Defects in homologous recombination DNA repair have become important predictive and prognostic factors in high-grade serous ovarian cancers. Emerging data indicate a potential role for evaluating homologous recombination deficiencies in uterine serous carcinoma. These deficiencies include mutations in associated genes (BRCA1, BRCA2), as well as diminished homologous recombination functional capacity and BRCA-associated genomic scars (homologous recombination deficient phenotype). A study of tumor samples found that $24 \%$ of endometrial cancers had homologous recombination deficiency. ${ }^{28}$ All these deficient tumors were nonendometrioid (half were uterine serous carcinoma and half carcinosarcoma with a serous component). When considering only tumors with a serous component, $67 \%$ of samples demonstrated homologous recombination deficiency. Wallbillich et al evaluated somatic genomic profile data of 451 patients with uterine serous carcinoma. ${ }^{29}$ Looking specifically at genetic mutations associated with homologous recombination, they found $16.9 \%$ had at least one mutation in the homologous recombination pathway. The most common of these was BRCA2. Taken together, these data support the clinical investigation of agents targeting tumors with homologous recombination deficiency, such as poly(ADP-ribose) polymerase inhibitors.

\section{Treatment}

Uterine serous carcinoma is surgically staged and is likely to present with metastatic disease at diagnosis. ${ }^{30}$ In an International Federation of Gynecology and Obstetrics (FIGO) annual report, stage II-IV disease was noted at presentation in $46 \%$ of women with uterine serous carcinoma compared with $21 \%$ of women with endometrioid adenocarcinoma. ${ }^{30}$ The surgical pathologic protocol, Gynecologic Oncology Group (GOG) 210 provides a large, contemporary dataset of uterine serous carcinoma tumors removed at the time of hysterectomy with surgical staging. ${ }^{31}$ Lymph node status remains among the most important prognostic factors in uterine serous carcinoma. Notably, the risk of pelvic nodal metastasis in GOG 210 was nearly $8 \%$ for non-invasive uterine serous carcinoma tumors, $18 \%$ for minimally invasive ( $<50 \%$ myometrial invasion), and $44 \%$ for deeply invasive tumors. Similarly, rates of metastatic involvement of aortic nodes were $4.2 \%, 11.3 \%$, and $32.4 \%$, respectively, for women with non-invasive, minimally invasive, and deeply invasive tumors, highlighting the importance of a thorough lymph node assessment in these settings.

\section{Early-Stage Disease}

Surgery

For women with apparent uterine confined disease, a total hysterectomy, bilateral salpingo-oophorectomy, pelvic washings, pelvic and aortic lymphadenectomy with or without omentectomy is recommended. Nodal assessment using variables often associated with low-grade tumors, is not reliable for assessing metastatic disease in uterine serous carcinoma. Additionally, patients with uterine serous carcinoma confined to the uterus were still at a significant risk for recurrence ( $21 \%$ overall) and poor survival outcomes, regardless of the presence of risk factors such as lymphovascular invasion, tumor size, or percentage of uterine serous carcinoma histology in the uterine specimens. ${ }^{32}$

Minimally invasive hysterectomy and staging is considered the standard surgical approach for women with endometrial cancer. ${ }^{33}$ For uterine serous carcinoma, minimally invasive techniques are acceptable when disease is clinically confined to the uterus. An ancillary analysis of more than 700 patients with high-grade (including uterine serous carcinoma) apparent early-stage endometrial cancer from the Gynecologic Oncology Group LAP2 trial as well as a multisite, retrospective cohort study of 383 similar patients. both demonstrated that women staged by minimally invasive techniques had fewer complications than, and similar survival outcomes to, those staged by laparotomy. ${ }^{34}$ Minimization of surgical morbidity is of interest as this population is often elderly and most will require adjuvant therapies. A minimally invasive approach is reasonable when extra-uterine disease is not evident on pre-operative imaging. Although many gynecologic oncologists perform omentectomy as part of the staging procedure, Gehrig et al demonstrated that omental sampling may not be needed in the routine surgical staging of uterine serous carcinoma as a grossly normal omentum was microscopically positive in $4 \%$ of cases reviewed. The sensitivity of a visually negative omentum was 0.89 $(p<0.0001){ }^{36}$ The addition of omentectomy in apparent stage I patients was recently found to have no impact on overall survival in a large, national database study. ${ }^{37}$

Lymphadenectomy in apparent early-stage uterine serous carcinoma is important. The retrospective, multisite cohort SEPAL study of more than 400 women with newly diagnosed, apparent earlystage endometrial cancer at high intermediate risk of recurrence (including uterine serous carcinoma) demonstrated that overall survival was significantly longer in the pelvic and para-aortic lymphadenectomy group than in the pelvic lymphadenectomy group (HR $0.53,95 \% \mathrm{Cl} 0.38$ to 0.76 ) and that this survival effect remained after controlling for post-operative chemotherapy. ${ }^{38}$ The updated National Comprehensive Cancer Network (NCCN) guidelines for treatment of uterine cancers now recognize sentinel lymph node (SLN) mapping as an alternative standard to systematic lymphadenectomy. In a prospective trial evaluating the characteristics of SLN mapping in high-grade endometrial cancers $(30 \%$ of which were uterine serous carcinoma), Soliman et al enrolled 101 patients who were managed using a prespecified protocol, including side-specific lymphadenectomy when no sentinel node was identified. ${ }^{39}$ The study team reported a sensitivity of $95 \%$ and if side-specific lymphadenectomy was performed when a SLN was not detected the false-negative rate was $4.3 \%$. A recent retrospective report of 245 patients with uterine serous carcinoma demonstrated similar 2-year survival outcomes for those women 
who underwent comprehensive lymphadenectomy and those who underwent SLN biopsy. ${ }^{40}$ This report is novel and included a relatively large sample size of patients with uterine serous carcinoma, but was not powered to determine the impact on survival based on surgical staging approach. Finally, the Canadian SENTOR trial enrolled 156 patients with high-grade, apparent early-stage endometrial cancer who underwent SLN biopsy followed by comprehensive pelvic and aortic lymphadenectomy. ${ }^{41}$ SLN detection rates were $99 \%$ per patient, with $77 \%$ detected bilaterally. Of 26 patients $(17 \%)$ with nodal metastases, 25 were identified by the SLN algorithm, for a patient-specific sensitivity of $96 \%(95 \% \mathrm{Cl} 80 \%$ to $100 \%)$, false-negative rate of $3.9 \%$ (95\% Cl $0 \%$ to $19 \%)$, and negative predictive value of $99 \%$ (95\% Cl $96 \%$ to $100 \%)$. The authors concluded that randomized data are needed to ascertain the impact of sentinel lymph node biopsy on survival in this patient population. Given the propensity for lymph node involvement in women with uterine serous carcinoma, a comprehensive lymph node assessment strategy, whether by conventional or SLN techniques, and evaluation of the pelvic and aortic lymph node basins, is recommended until further data are available.

\section{Adjuvant Therapy}

Prospective data regarding the role of adjuvant therapy in earlystage uterine serous carcinoma are limited. Women with uterine serous carcinoma were excluded from GOG protocol 99, allowed in PORTEC-1 (however, few enrolled), and excluded from PORTEC-2. Both ASTEC and GOG 249 allowed enrollment of women with earlystage uterine serous carcinoma, representing $10 \%$ and $15 \%$ of the overall study populations, respectively. ${ }^{42}{ }^{43}$ Consequently these studies were underpowered to detect survival differences by treatment type in patients with uterine serous carcinoma. Thus, retrospective evaluations of large, population-based databases provide the best evidence with which to guide our understanding of adjuvant therapy for early-stage disease.

Both the high rates of recurrence in uterine serous carcinoma and the propensity for a recurrence to be extrapelvic and multisite have supported a rationale for systemic chemotherapy. In one retrospective multi-institutional series, 142 patients with stage I uterine serous carcinoma who had undergone observation, radiation, or chemotherapy (with or without vaginal brachytherapy) after hysterectomy and staging surgery were compared. ${ }^{44}$ Twenty-five recurrences (17.6\%) were diagnosed, and $60 \%$ were extrapelvic. Patients treated with chemotherapy had fewer recurrences (11.2\%) than patients who received radiation alone $(25 \%, p=0.146)$ or observation $(30.3 \%, p=0.016)$. This effect was most pronounced in the patients with myometrial invasion $(p=0.007)$. After multivariate analysis, treatment with chemotherapy was associated with a decreased risk of recurrence $(p=0.047)$ and progressionfree survival also significantly favored the chemotherapy-treated patients $(p=0.013)$.

Cham et al used the National Cancer Database (NCDB) to evaluate post-operative adjuvant therapy in 7325 patients with stage I-II uterine serous cancers. ${ }^{45}$ Use of chemotherapy was associated with a $22 \%$ reduction in mortality $(\mathrm{HR} 0.78,95 \% \mathrm{Cl} 0.69$ to 0.80 ), and post-operative vaginal brachytherapy a $33 \%$ reduction in mortality (HR $0.67,95 \% \mathrm{Cl} 0.57$ to 0.78 ). Notably, pelvic external beam radiotherapy was not associated with a benefit in this population. In a subset analysis by stage, chemotherapy was beneficial in stage IB-II cancers and vaginal brachytherapy was beneficial in both stage IA and II cancers.

Hong et al also used the NCDB to evaluate 5432 women with stage I uterine serous carcinoma. ${ }^{46}$ The best overall survival estimates were associated with the combination of chemotherapy and radiation, regardless of the completeness of surgical staging. Multivariable analysis demonstrated a survival benefit for surgically staged patients who received chemotherapy (HR $0.77,95 \% \mathrm{Cl}$ 0.64 to 0.94$)$ and vaginal brachytherapy $(\mathrm{HR} 0.68,95 \% \mathrm{Cl} 0.53$ to 0.88 ). Among patients without a lymphadenectomy, receipt of vaginal brachytherapy was associated with a significant survival advantage.

Another NCDB study evaluated patients who would have met criteria for GOG 249 and were treated with either chemotherapy plus vaginal brachytherapy or pelvic external beam radiotherapy. ${ }^{47}$ Women with uterine serous carcinoma who were treated with external beam radiotherapy alone experienced a significantly increased risk of death (HR 1.76, $95 \% \mathrm{Cl} 1.18$ to 2.64) compared with those treated with chemotherapy and vaginal brachytherapy.

A retrospective analysis of patients enrolled in PORTEC-3, which included patients with early-stage uterine serous carcinoma randomized to chemoradiation versus radiation alone, noted significant improvements in both recurrence-free survival and overall survival with combination therapy. Specifically, tumors characterized by abnormal p53 expression (a common feature of uterine serous carcinoma) benefited from chemoradiation compared with radiation alone with recurrence-free survival of $58.6 \%$ vs $36.2 \%$ ( $\mathrm{HR} 0.52,95 \% \mathrm{Cl} 0.3$ to $0.91, \mathrm{p}=0.02$ ) and overall survival of $64.9 \%$ vs $41.8 \%$ ( $\mathrm{HR} 0.55,95 \% \mathrm{Cl} 0.30$ to $1, \mathrm{p}=0.49) .{ }^{48}$

\section{Recommendations}

In accordance with international guidelines, ${ }^{49}{ }^{50}$ we recommend six cycles of intravenous carboplatin and paclitaxel and consideration of brachytherapy for the treatment of stage I disease, and this same multimodal regimen or chemotherapy and external beam radiotherapy plus vaginal brachytherapy, for select patients with stage II disease. It is not clear whether patients with uterine serous carcinoma confined to a uterine polyp benefit from chemotherapy, but it is our practice to recommend chemotherapy for patients with any residual disease in the uterus at hysterectomy (Table 1).

\section{Advanced Stage Disease}

Surgery

An estimated $50-60 \%$ of patients with uterine serous carcinoma present with stage III or IV disease. ${ }^{3}$ Women with extra-uterine disease at diagnosis are surgically managed and a comprehensive lymph node assessment should be performed as discussed for early-stage disease. Identification and removal of bulky lymph nodes or bulky implants/masses is recommended. Several retrospective studies suggest cytoreductive surgery may confer a survival benefit in women with metastatic uterine serous carcinoma. In a report of 70 women with stages IIIC or IV uterine serous carcinoma, a significant difference in median time to recurrence (9 vs 6 months, $p=0.04$ ) and median survival ( 20 vs 12 months, $p=0.02$ ) was observed between optimally and suboptimally cytoreduced patients. ${ }^{51}$ Therefore, in those with advanced stage disease, optimal cytoreduction-ideally a complete gross resection-is an integral component of surgical treatment. For patients with disease 
Table 1 Summary recommendations for post-operative therapy

\begin{tabular}{|c|c|c|c|}
\hline Disease setting & Target & Adjuvant therapy & Level of evidence \\
\hline \multirow{2}{*}{$\begin{array}{l}\text { Early stage } \\
\text { Including disease } \\
\text { confined to a polyp at } \\
\text { hysterectomy }\end{array}$} & \multirow[t]{2}{*}{ ND } & Chemotherapy* (preferred) & \\
\hline & & VBT & Retrospective, population-based data \\
\hline \multirow[t]{3}{*}{ Advanced stage } & HER2+ & Chemotherapy*+trastuzumab & Prospective, randomized data \\
\hline & \multirow[t]{2}{*}{ ND and HER2- } & Chemotherapy* & Prospective, randomized data \\
\hline & & EBRT for selected cases & Retrospective, population-based data \\
\hline \multirow[t]{6}{*}{ Recurrent disease } & \multirow[t]{2}{*}{ HER2+ } & Chemotherapy*+trastuzumab & \multirow{6}{*}{ Prospective, randomized data } \\
\hline & & Adavosertib & \\
\hline & \multirow[t]{2}{*}{ HER2- } & Chemotherapy & \\
\hline & & Adavosertib & \\
\hline & $\mathrm{MSI}$ & Pembrolizumab & \\
\hline & MSS & $\begin{array}{l}\text { Pembrolizumab+ } \\
\text { Lenvatinib }\end{array}$ & \\
\hline
\end{tabular}

${ }^{*}$ Doublet of paclitaxel and carboplatin.

EBRT, external beam radiotherapy; MSI,

microsatellite instable; MSS, microsatellite stable; ND, not defined; VBT, vaginal brachytherapy.

that is unresectable at presentation, neo-adjuvant chemotherapy is offered. If there is a substantial response to systemic therapy, a delayed surgical effort should be considered. ${ }^{50}$

\section{Adjuvant Therapy}

With the landmark GOG 122 study evaluating whole abdominal radiation compared with chemotherapy in advanced endometrial cancers (approximately $20 \%$ of patients with uterine serous carcinoma), chemotherapy has been viewed as the standard adjuvant treatment for high-risk disease. ${ }^{52}$ Several chemotherapeutic agents are active, with the combination of paclitaxel and carboplatin being preferred. ${ }^{49} 53$ Contemporary post-surgical management of advanced-stage disease is stratified by whether the uterine serous carcinoma tumors are HER2 negative or positive.

\section{HER2-Negative Disease}

The available randomized evidence for the adjuvant management of high-risk endometrial cancers without a molecular classifier is conflicting. The phase III PORTEC-3 trial included 105 women with stages IA-III uterine serous carcinoma, which comprised $16 \%$ of the total study population. ${ }^{54}$ Patients were randomly assigned to chemoradiotherapy with cisplatin during pelvic external beam radiotherapy followed by systemic paclitaxel and carboplatin for four additional cycles versus pelvic external beam radiotherapy alone. The trial evaluated 660 women and met the primary overall survival endpoint. Adjusted 5-year overall survival for the entire cohort was $81.4 \%$ for the chemoradiotherapy group versus $76.6 \%$ for the external beam radiotherapy group (HR $0.70,95 \% \mathrm{Cl} 0.51$ to 0.97$)$. Notably, women with uterine serous carcinoma were more likely to have a recurrence of disease and more likely to have distant metastatic disease at recurrence-contributing to worse overall survival compared with other subtypes. In an exploratory post hoc analysis, patients with uterine serous carcinoma derived significant survival benefit from chemoradiotherapy with a 5 -year overall survival of $71.4 \%$ vs $52.8 \%$ for external beam radiotherapy alone (HR $0.48,95 \% \mathrm{Cl} 0.24$ to 0.96$)$. The results of
PORTEC-3 demonstrate that external beam radiotherapy alone is insufficient for the management of high-risk endometrial cancers in this setting.

The positive findings of PORTEC-3 are in contrast to GOG 258, which evaluated high risk endometrial cancers by randomizing patients to receive carboplatin and paclitaxel chemotherapy alone versus chemoradiotherapy, which was given as described for PORTEC- $3{ }^{55}$ The protocol enrolled patients with stage IA-II uterine serous carcinoma with positive washings $(<3 \%$ of the total population) as well as patients with stage III-IVa disease. Patients with uterine serous carcinoma made up $18 \%(131 / 736)$ of the total study population. The primary endpoint was relapse-free survival, and no significant difference was observed between to the two treatment arms: $59 \%$ for the chemoradiotherapy group versus $58 \%$ for the chemotherapy only group ( $\mathrm{HR} 0.90,90 \% \mathrm{Cl}, 0.74$ to 1.10 ). Chemoradiotherapy was associated with significantly lower vaginal and nodal recurrences, but a higher rate of distant recurrence was observed. Subgroup analyses, including an examination of patients with uterine serous carcinoma, failed to identify any factors that would favor chemoradiotherapy over chemotherapy. The authors concluded that the addition of radiation did not provide a relapsefree survival benefit over chemotherapy alone in the setting of highrisk endometrial cancer.

When multimodal therapy is used, questions remain regarding the sequencing of chemotherapy and radiation. Single-institution data with a large population of women with advanced uterine serous carcinoma tumors and long follow-up suggested an improvement in 5-year overall survival when external beam radiotherapy was given after chemotherapy. ${ }^{56}$ In a populationbased analysis of 2902 patients with uterine serous carcinoma with stage IIIC disease, Lin et al noted that patients treated with chemotherapy followed by external beam radiotherapy were $31 \%$ less likely to die (HR $0.69,95 \% \mathrm{Cl} 0.56$ to 0.84 ) than those treated with chemotherapy alone. ${ }^{57}$ 
HER2-Positive Disease

Trastuzumab, a monoclonal antibody directed at HER2, was the first drug approved by the US Food and Drug Administration (FDA) in 1998 for treating HER2-positive metastatic breast cancer. Five additional HER2 targeting agents (including monoclonal antibodies, antibody drug conjugates and oral small molecule tyrosine kinase inhibitors) have now been approved by the FDA which have revolutionized the approach to HER2-positive breast cancer and other solid tumor malignancies. ${ }^{24}$

In a randomized phase II trial, Fader et al evaluated 61 patients with advanced (stage III-IV) or recurrent, HER2-positive uterine serous carcinoma. ${ }^{58}$ Patients received carboplatin and paclitaxel with or without trastuzumab followed by trastuzumab maintenance, continued until disease progression or prohibitive toxicity. In the overall cohort, patients who received trastuzumab had an improved median progression-free survival of 12.6 vs 8.0 months (HR 0.44 , $95 \% \mathrm{Cl} 0.26$ to $0.76 ; p=0.005)$. However, patients with stage IIII-IV disease treated in the primary setting demonstrated the greatest benefit from trastuzumab with a progression-free survival of 17.9 vs 9.3 months ( $\mathrm{HR} 0.40,90 \% \mathrm{Cl} 0.20$ to $0.80 ; \mathrm{p}-0.013$ ). A recently updated survival analysis also showed a significant improvement of 5.2 months in median overall survival in women who received trastuzumab ( $\mathrm{HR} 0.58,95 \% \mathrm{Cl} 0.34$ to $0.99 ; \mathrm{p}=0.046$ ), again with the greatest benefit observed in those patients with advanced-stage disease treated in the primary setting. ${ }^{59}$ The benefit was most notable in those with stage III-IV disease, with survival median not reached in the trastuzumab arm versus 24.4 months in the control arm (HR $0.49,90 \% \mathrm{Cl} 0.25$ to $0.97 ; \mathrm{p}=0.041)$. Importantly, toxicity was not significantly different with the addition of the targeted therapy. These encouraging results prompted changes in the NCCN uterine cancer guidelines, such that trastuzumab in addition to platinum and taxane-based chemotherapy is the preferred regimen for women with advanced or recurrent HER2-positive uterine serous carcinoma.

Trial development is underway to test the efficacy of newer HER2 targeting agents in uterine serous carcinoma. Afatinib, an oral HER2 tyrosine kinase inhibitor, has demonstrated strong antitumor effects in uterine serous carcinoma cell lines. ${ }^{60}$ Therefore, it is being actively studied in women with HER2-positive uterine serous carcinoma and results are forthcoming (NCT 02491099). ${ }^{61}$ Additionally, clinical trial development with antibody drug conjugates and with dual HER2 inhibition antibody drug conjugates are ongoing. Specifically, NRG Oncology GY-026, an international three-arm, phase III study of women with primary stage I-IV, HER2-positive disease examining the role of mono (trastuzumab) or dual inhibition of HER2 (trastuzumab/pertuzumab) in addition to cytotoxic chemotherapy will open soon.

\section{Recommendations}

We believe patients should be enrolled in a clinical trial if available (Table 2). Outside of a clinical trial, adjuvant therapy for advanced-stage disease should be determined based on HER2 status of the tumor. For HER2-negative cancers, the results of GOG 122, GOG 258, and PORTEC-3 demonstrate that platinum-based chemotherapy is a fundamental aspect of treatment. The benefit of external beam radiotherapy is less clear, and the results of the largest trial addressing this question (GOG 258) suggest that there is no relapse-free survival benefit from adding radiation. We recommend an attempt at maximal cytoreductive surgery followed by six cycles of carboplatin and paclitaxel in this setting, with post-chemotherapy external beam radiotherapy evaluated on an individual basis. For HER2-positive, advanced-stage cancers, we recommend an attempt at maximal cytoreductive surgery followed by six cycles of trastuzumab plus paclitaxel and carboplatin, followed by trastuzumab maintenance (Table 1).

\section{Recurrent Disease}

Immune Checkpoint Inhibition in Uterine Serous Carcinoma Although immunotherapy is emerging as an exciting new treatment option for women with uterine cancer, uterine serous carcinoma seems to be less immunogenic than other uterine cancer subtypes. Biomarkers that often predict response to

Table 2 Active clinical trials of targeted therapy in uterine serous carcinoma

\begin{tabular}{llll}
\hline NCT number & Phase & Molecular target & Investigational agent \\
\hline 03552471 & I & FR- $\alpha$ & Reference \\
03120624 & PARP & Mirvetuximab & Rucaparib \\
02142803 & I & IFN- $\beta$ receptor & IFN- $\beta$ by viral vector \\
01935973 & mTORC & MLN0128 & 70 \\
01935934 & I & VEGF & Bevacizumab \\
04080284 & MEK & Trametinib \\
03668340 & II & AKT & GSK2141795 \\
02874430 & II & VEGF & Cabozantinib \\
02491099 & II & PARP & Niraparib \\
03914612 & II & WEE1 & Adavosertib \\
\hline II & AMPK & Metformin & 72 \\
\hline
\end{tabular}

AKT also known as protein kinase $B$.

AMPK, AMP-activated protein kinase; FR, folate receptor; IFN, Interferon; MEK, mitogen-activated protein kinase; mTORC, mammalian target of rapamycin; PARP, poly(ADP-ribose) polymerase; PDL-1, programed death ligand 1; VEGF, vascular endothelial growth factor. 
immunotherapy-microsatellite instability, and PDL1 expressionare not frequently seen in uterine serous carcinoma. ${ }^{62}$ However, in the Keynote-146 trial, women with recurrent uterine serous carcinoma showed a remarkable response to the combination of pembrolizumab (a PD-1 inhibitor) and lenvatinib, a multi-kinase inhibitor. Of the 33 women with uterine serous carcinoma enrolled in this trial, half had either a partial or complete response to therapy, which is an unprecedented outcome in this patient population. ${ }^{63}$ Data from Keynote 775 was recently reported by Makker et al. ${ }^{64}$ In this phase III study, patients (regardless of mismatch repair status) were randomized to either pembrolizumab with lenvatinib or physician's choice of chemotherapy. Progression-free survival was significantly improved with pembrolizumab and lenvatinib (7.2 vs 3.8 months; HR 0.56 ) and overall survival was also significantly improved (18.3 vs 11.4 months; HR 0.62). Importantly, clinical benefit was seen in patients with proficient mismatch repair, which includes uterine serous carcinoma.

To further test the efficacy of immunotherapy in endometrial cancer, regardless of biomarker status, a large cooperative group trial is underway, which is testing the addition of pembrolizumab to standard chemotherapy (NCT 03914612). ${ }^{65}$ Over 800 patients will be enrolled and one-third will probably be patients with uterine serous carcinoma. This trial will provide valuable information regarding the addition of immunotherapy via checkpoint inhibition in women with advance uterine cancer, including uterine serous carcinoma.

\section{WEE1 Inhibition}

Adavosertib is a relatively new anticancer therapy that targets WEE1, a protein tyrosine kinase involved in cell cycle checkpoints. Inhibiting this protein leads to unstable DNA replication structures, which ultimately causes lethal cell damage. Cancers with TP53 mutations, such as uterine serous carcinoma, may be more likely to respond to WEE1 inhibition because they already have high levels of replication stress and endogenous DNA damage. In a recent phase II study of 27 women with recurrent uterine serous carcinoma, the overall response rate to this therapy was nearly $30 \%$, although side effects were significant (including anemia, gastrointestinal symptoms, and fatigue) ${ }^{66} \mathrm{~A}$ larger phase II study of over 100 women with uterine serous carcinoma is planned to verify these results.

\section{Other Targets}

Given the aforementioned emerging data demonstrating a significant rate of homologous recombination deficiency in uterine serous carcinoma, therapeutic interest in poly(ADP-ribose) polymerase inhibitors is understandable. Prospective clinical data in uterine serous carcinoma are limited. Currently, a trial of niraparib in the maintenance setting is enrolling patients with uterine serous carcinoma (NCT 04080284). ${ }^{67}$

The phosphoinositide 3-kinase/AKT pathway is active in many endometrial cancers, and a small number of patients with uterine serous carcinoma have been studied with AKT inhibitors. An exploratory analysis of a recent phase II study of MK-2206 demonstrated that patients with uterine serous carcinoma had an improved 6-month progression free-survival compared with other histologies. $^{68}$

\section{CONCLUSION}

Despite the poor prognosis of uterine serous carcinoma, recent advances in molecular assessment and treatment of this disease have demonstrated significant improvements in survival outcomes in both the upfront and recurrent settings. Accurate surgical staging, with aggressive cytoreduction when indicated, remains the most critical step in treatment. Equally important, the correct pathologic and molecular classification of uterine serous cancers sets the stage for proper systemic adjuvant therapies to be prescribed. Therapeutic targeting of HER2 is among the most significant advancements in this disease in decades. Emerging opportunities with combinations of immunotherapies and tyrosine kinase inhibitors as well as other targets such as WEE1 are exciting. These advancements will allow us to provide effective treatments for a population of women who have carried the disproportionate burden of this disease.

\section{Twitter Amanda N Fader @amandanfader}

Contributors All authors made substantial contributions to the conception, drafting, critical revision, and have given their approval of the manuscript. All authors agree to be accountable for all aspects of the work.

Funding The authors have not declared a specific grant for this research from any funding agency in the public, commercial or not-for-profit sectors.

Competing interests None declared.

Patient consent for publication Not required.

Provenance and peer review Not commissioned; externally peer reviewed.

ORCID iD

J Stuart Ferriss http://orcid.org/0000-0002-2053-8604

\section{REFERENCES}

1 Siegel RL, Miller KD, Jemal A. Cancer statistics, 2020. CA Cancer J Clin 2020;70:7-30.

2 Henley SJ, Miller JW, Dowling NF, et al. Uterine cancer incidence and mortality - United States, 1999-2016. MMWR Morb Mortal Wkly Rep 2018;67:1333-8.

3 Hamilton CA, Cheung MK, Osann K, et al. Uterine papillary serous and clear cell carcinomas predict for poorer survival compared to grade 3 endometrioid corpus cancers. Br J Cancer 2006;94:642-6.

4 Clement PB, Young RH. Non-endometrioid carcinomas of the uterine corpus: a review of their pathology with emphasis on recent advances and problematic aspects. Adv Anat Pathol 2004;11:117-42.

5 Bregar AJ, Alejandro Rauh-Hain J, Spencer R, et al. Disparities in receipt of care for high-grade endometrial cancer: a national cancer data base analysis. Gynecol Oncol 2017;145:114-21.

6 Fisher B, Costantino JP, Redmond CK, et al. Endometrial cancer in tamoxifen-treated breast cancer patients: findings from the National Surgical Adjuvant Breast and Bowel Project (NSABP) B-14. J Natl Cancer Inst 1994;86:527-37.

7 Gehrig PA, Bae-Jump VL, Boggess JF, et al. Association between uterine serous carcinoma and breast cancer. Gynecol Oncol 2004;94:208-11.

8 Bland AE, Calingaert B, Secord AA, et al. Relationship between tamoxifen use and high risk endometrial cancer histologic types. Gynecol Oncol 2009;112:150-4.

9 Hornreich G, Beller U, Lavie O, et al. Is uterine serous papillary carcinoma a BRCA1-related disease? Case report and review of the literature. Gynecol Oncol 1999;75:300-4.

10 Lavie O, Hornreich G, Ben Arie A, et al. BRCA1 germline mutations in women with uterine serous papillary carcinoma. Obstet Gynecol 2000;96:28-32.

11 Lavie O, Hornreich G, Ben-Arie A, et al. BRCA germline mutations in Jewish women with uterine serous papillary carcinoma. Gynecol Oncol 2004;92:521-4.

12 Lavie O, Ben-Arie A, Segev Y, et al. BRCA germline mutations in women with uterine serous carcinoma--still a debate. Int J Gynecol Cancer 2010;20:1531-4. 
13 Pennington KP, Walsh T, Lee M, et al. BRCA1, TP53, and CHEK2 germline mutations in uterine serous carcinoma. Cancer 2013;119:332-8.

14 Shu CA, Pike MC, Jotwani AR, et al. Uterine cancer after riskreducing salpingo-oophorectomy without hysterectomy in women with BRCA mutations. JAMA Oncol 2016;2:1434-40.

15 Laitman Y, Michaelson-Cohen R, Levi E, et al. Uterine cancer in Jewish Israeli BRCA1/2 mutation carriers. Cancer 2019;125:698-703.

16 Yemelyanova A, Ji H, Shih I-M, et al. Utility of p16 expression for distinction of uterine serous carcinomas from endometrial endometrioid and endocervical adenocarcinomas: immunohistochemical analysis of 201 cases. Am J Surg Pathol 2009;33:1504-14.

17 Boyraz G, Salman MC, Basaran D, et al. Extrauterine spread, adjuvant treatment, and prognosis in noninvasive uterine papillary serous carcinoma of the endometrium: a retrospective multicenter study. Int J Gynecol Cancer 2017;27:102-8.

18 Kuhn E, Wu R-C, Guan B, et al. Identification of molecular pathway aberrations in uterine serous carcinoma by genome-wide analyses. $J$ Natl Cancer Inst 2012;104:1503-13.

19 Kuhn E, Bahadirli-Talbott A, Shih I-M. Frequent CCNE1 amplification in endometrial intraepithelial carcinoma and uterine serous carcinoma. Mod Pathol 2014;27:1014-9.

20 Cancer Genome Atlas Research Network, Kandoth C, Schultz N, et al. Integrated genomic characterization of endometrial carcinoma. Nature 2013;497:67-73.

21 Talhouk A, McConechy MK, Leung S, et al. Confirmation of ProMisE: a simple, genomics-based clinical classifier for endometrial cancer. Cancer 2017;123:802-13.

22 Talhouk A, Derocher H, Schmidt P, et al. Molecular subtype not immune response drives outcomes in endometrial carcinoma. Clin Cancer Res 2019;25:2537-48.

23 Casalini P, Iorio MV, Galmozzi E, et al. Role of HER receptors family in development and differentiation. J Cell Physiol 2004;200:343-50.

24 Erickson BK, Zeybek B, Santin AD, et al. Targeting human epidermal growth factor receptor 2 (HER2) in gynecologic malignancies. Curr Opin Obstet Gynecol 2020;32:57-64.

25 Erickson BK, Najjar O, Damast S, et al. Human epidermal growth factor 2 (HER2) in early stage uterine serous carcinoma: a multiinstitutional cohort study. Gynecol Oncol 2020;159:17-22.

26 Wolff AC, Hammond MEH, Allison KH, et al. Human epidermal growth factor receptor 2 testing in breast cancer: American Society of Clinical Oncology/College of American Pathologists clinical practice guideline focused update. J Clin Oncol 2018;36:2105-22.

27 Banet N, Shahi M, Batista D, et al. HER-2 amplification in uterine serous carcinoma and serous endometrial intraepithelial carcinoma. Am J Surg Pathol 2021;45:708-15.

28 de Jonge MM, Auguste A, van Wijk LM, et al. Frequent homologous recombination deficiency in high-grade endometrial carcinomas. Clin Cancer Res 2019;25:1087-97.

29 Wallbillich JJ, Morris RT, Ali-Fehmi R. Comparing mutation frequencies for homologous recombination genes in uterine serous and high-grade serous ovarian carcinomas: a case for homologous recombination deficiency testing in uterine serous carcinoma. Gynecol Oncol 2020;159:381-6.

30 Mhawech-Fauceglia P, Herrmann RF, Kesterson J, et al. Prognostic factors in stages II/III/IV and stages III/IV endometrioid and serous adenocarcinoma of the endometrium. Eur J Surg Oncol 2010;36:1195-201.

31 Creasman WT, Ali S, Mutch DG, et al. Surgical-pathological findings in type 1 and 2 endometrial cancer: an NRG Oncology/Gynecologic Oncology Group study on GOG-210 protocol. Gynecol Oncol 2017;145:519-25.

32 Fader AN, Starks D, Gehrig PA, et al. An updated clinicopathologic study of early-stage uterine papillary serous carcinoma (UPSC). Gynecol Oncol 2009;115:244-8.

33 Walker JL, Piedmonte MR, Spirtos NM, et al. Laparoscopy compared with laparotomy for comprehensive surgical staging of uterine cancer: Gynecologic Oncology Group study LAP2. J Clin Oncol 2009;27:5331-6.

34 Fader AN, Java J, Tenney M, et al. Impact of histology and surgical approach on survival among women with early-stage, high-grade uterine cancer: an NRG Oncology/Gynecologic Oncology Group ancillary analysis. Gynecol Oncol 2016;143:460-5.

35 Fader AN, Seamon LG, Escobar PF, et al. Minimally invasive surgery versus laparotomy in women with high grade endometrial cancer: a multi-site study performed at high volume cancer centers. Gynecol Oncol 2012;126:180-5.
36 Gehrig PA, Van Le L, Fowler WC. The role of omentectomy during the surgical staging of uterine serous carcinoma. Int $J$ Gynecol Cancer 2003;13:212-5.

37 Nasioudis D, Heyward Q, Gysler S, et al. Is there a benefit of performing an omentectomy for clinical stage I high-grade endometrial carcinoma? Surg Oncol 2021;37:101534.

38 Todo $\mathrm{Y}$, Kato H, Kaneuchi M, et al. Survival effect of para-aortic lymphadenectomy in endometrial cancer (SEPAL study): a retrospective cohort analysis. Lancet 2010;375:1165-72.

39 Soliman PT, Westin SN, Dioun S, et al. A prospective validation study of sentinel lymph node mapping for high-risk endometrial cancer. Gynecol Oncol 2017;146:234-9.

40 Basaran D, Bruce S, Aviki EM, et al. Sentinel lymph node mapping alone compared to more extensive lymphadenectomy in patients with uterine serous carcinoma. Gynecol Oncol 2020;156:70-6.

41 Cusimano MC, Vicus D, Pulman K, et al. Assessment of sentinel lymph node biopsy vs lymphadenectomy for intermediate- and highgrade endometrial cancer staging. JAMA Surg 2021;156:157-64.

42 ASTEC/EN.5 Study Group, Blake P, Swart AM, et al. Adjuvant external beam radiotherapy in the treatment of endometrial cancer (MRC ASTEC and NCIC CTG EN.5 randomised trials): pooled trial results, systematic review, and meta-analysis. Lancet 2009;373:137-46.

43 Randall ME, Filiaci V, McMeekin DS, et al. Phase III trial: adjuvant pelvic radiation therapy versus vaginal brachytherapy plus paclitaxel/carboplatin in high-intermediate and high-risk early stage endometrial cancer. J Clin Oncol 2019;37:1810-8.

44 Fader AN, Drake RD, O'Malley DM, et al. Platinum/taxane-based chemotherapy with or without radiation therapy favorably impacts survival outcomes in stage I uterine papillary serous carcinoma. Cancer 2009;115:2119-27.

45 Cham S, Huang Y, Tergas Al, et al. Utility of radiation therapy for early-stage uterine papillary serous carcinoma. Gynecol Oncol 2017;145:269-76.

46 Hong JC, Foote J, Broadwater G, et al. Impact of chemotherapy and radiotherapy on management of early stage clear cell and papillary serous carcinoma of the uterus. Int J Gynecol Cancer 2017;27:720-9.

47 Tatebe K, Hasan Y, Son $\mathrm{CH}$. Adjuvant vaginal brachytherapy and chemotherapy versus pelvic radiotherapy in early-stage endometrial cancer: outcomes by risk factors. Gynecol Oncol 2019;155:429-35.

48 León-Castillo A, de Boer SM, Powell ME, et al. Molecular classification of the PORTEC-3 trial for high-risk endometrial cancer: impact on prognosis and benefit from adjuvant therapy. J Clin Oncol 2020;38:3388-97.

49 Koh W-J, Abu-Rustum NR, Bean S, et al. Uterine neoplasms, version 1.2018, NCCN clinical practice guidelines in oncology. J Natl Compr Canc Netw 2018;16:170-99.

50 Concin N, Ray-Coquard I, Glasspool RM, et al. European Network of Gynaecological Oncological Trial Groups' requirements for trials between academic groups and industry partners - a new model D for drug and medical device development. Int $J$ Gynecol Cancer 2020;30:730-4

51 Thomas MB, Mariani A, Cliby WA, et al. Role of cytoreduction in stage III and IV uterine papillary serous carcinoma. Gynecol Oncol 2007;107:190-3.

52 Randall ME, Filiaci VL, Muss $\mathrm{H}$, et al. Randomized phase III trial of whole-abdominal irradiation versus doxorubicin and cisplatin chemotherapy in advanced endometrial carcinoma: a Gynecologic Oncology Group study. J Clin Oncol 2006;24:36-44.

53 Miller D, Filiaci V, Fleming G, et al. Late-breaking abstract 1: randomized phase III noninferiority trial of first line chemotherapy for metastatic or recurrent endometrial carcinoma: a Gynecologic Oncology Group study. Gynecol Oncol 2012;125:771.

54 de Boer SM, Powell ME, Mileshkin L, et al. Adjuvant chemoradiotherapy versus radiotherapy alone in women with highrisk endometrial cancer (PORTEC-3): patterns of recurrence and post-hoc survival analysis of a randomised phase 3 trial. Lancet Oncol 2019;20:1273-85.

55 Matei D, Filiaci V, Randall ME, et al. Adjuvant chemotherapy plus radiation for locally advanced endometrial cancer. $N$ Engl J Med 2019;380:2317-26

56 Viswanathan AN, Macklin EA, Berkowitz R, et al. The importance of chemotherapy and radiation in uterine papillary serous carcinoma. Gynecol Oncol 2011;123:542-7.

57 Lin JF, Muñiz K, Sukumvanich P, et al. Survival advantage associated with multimodal therapy in women with node-positive (stage-IIIC) uterine papillary serous carcinoma: a National Cancer Database study. BJOG 2016;123:1846-52.

58 Fader AN, Roque DM, Siegel E, et al. Randomized phase II trial of carboplatin-paclitaxel versus carboplatin-paclitaxel-trastuzumab 


\section{Review}

in uterine serous carcinomas that overexpress human epidermal growth factor receptor 2/neu. J Clin Oncol 2018;36:2044-51.

59 Fader AN, Roque DM, Siegel E, eds. Randomized phase II trial of carboplatin-paclitaxel compared to carboplatin-paclitaxeltrastuzumab in advanced or recurrent uterine serous carcinomas that overexpress HER2/Neu (NCT01367002): Updated survival analysis. Toronto, CA: Society of Gynecologic Onoclogy Annual Meeting, 2020.

60 Schwab CL, Bellone S, English DP, et al. Afatinib demonstrates remarkable activity against HER2-amplified uterine serous endometrial cancer in vitro and in vivo. $\mathrm{Br} \mathrm{J}$ Cancer 2014:111:1750-6.

61 Santin AD. A phase II evaluation of afatinibin patients with persistent or recurrent HER2-positive uterine serous carcinoma (afatinib). NCT02491099. ClinicalTrials.gov: NIH. Available: https://clinicaltrials. gov/ct2/show/NCT02491099

62 Herzog TJ, Arguello D, Reddy SK, et al. PD-1, PD-L1 expression in 1599 gynecological cancers: implications for immunotherapy. Gynecol Oncol 2015;137:204-5.

63 Makker V, Taylor MH, Aghajanian C, et al. Lenvatinib plus pembrolizumab in patients with advanced endometrial cancer. $J$ Clin Oncol 2020;38:2981-92.

64 Makker V. Abstract 11512: A multicenter, open-label, randomized, phase III study to compare the efficacy and safety of lenvatinib in combination with pembrolizumab versus treatment of physician's choice in patients with advanced endometrial cancer. Society of Gynecologic Oncology Annual Meeting, 2021.

65 Eskander RN. Testing the addition of the immunotherapy drug pembrolizumab to the usual chemotherapy treatment (paclitaxel and carboplatin) in stage III-IV or recurrent endometrial cancer ClinicalTrials.gov: NIH. Available: https://clinicaltrials.gov/ct2/show/ NCT03914612
66 Liu JF, Xiong N, Campos SM, et al. Phase II study of the Wee1 inhibitor adavosertib in recurrent uterine serous carcinoma. $J$ Clin Oncol 2021;39:JCO2003167.

67 Frimer M. Trial of maintenance with niraparib- uterine serous carcinoma ClinicalTrials.gov: NIH. Available: https://clinicaltrials.gov/ ct2/show/NCT04080284

68 Myers AP, Konstantinopoulos PA, Barry WT, et al. Phase II, 2-stage, 2-arm, PIK3CA mutation stratified trial of MK-2206 in recurrent endometrial cancer. Int J Cancer 2020;147:413-22.

69 Backes F. Mirvetuximab soravtansine and rucaparib camsylate in treating participants with recurrent endometrial, ovarian, fallopian tube or primary peritoneal cancer: $\mathrm{NCl}$. Available: https:// clinicaltrials.gov/ct2/show/NCT03552471

70 Bakkum-Gamez JN. VSV-hIFNbeta-NIS with or without ruxolitinib phosphate in treating patients with stage IV or recurrent endometria cancer clinicalTrials.gov: NCl. Available: https://clinicaltrials.gov/ct2/ show/NCT03120624

71 Nayak L. TORC1/2 inhibitor MLN0128 and bevacizumab in treating patients with recurrent glioblastoma or advanced solid tumors: $\mathrm{NCl}$. Available: https://clinicaltrials.gov/ct2/show/NCT02142803

72 Westin SN. Trametinib with or without GSK2141795 in treating patients with recurrent or persistent endometrial cancer: $\mathrm{NCl}$. Available: https://clinicaltrials.gov/ct2/show/NCT01935973

73 Dhani N. Cabozantinib s-malate in treating patients with recurrent or metastatic endometrial cancer: $\mathrm{NCl}$. Available: https://clinicaltrials. gov/ct2/show/NCT01935934

74 Liu J. AZD1775 in women with recurrent or persistent uterine serous carcinoma or uterine carcinosarcoma: NCl. Available: https:// clinicaltrials.gov/ct2/show/NCT03668340

75 Johnson J. Metformin hydrochloride and doxycycline in treating patients with localized breast or uterine cancer: NCI. Available: https://clinicaltrials.gov/ct2/show/NCT02874430 